\title{
Lexicographic Quasilinear Utility
}

\author{
Yutaka Nakamura *
}

September 8, 2000

\begin{abstract}
This paper presents necessary and sufficient conditions for the existence of a finite-dimensional quasilinear utility function whose lexicographically ordered utility vectors preserve a decision maker's preference order on a mixture space.
\end{abstract}

\section{Introduction}

The aim of the paper is to identify and discuss axioms for a binary relation $\succ$ on a mixture space $\mathscr{M}$ that are necessary and sufficient for the existence of a finite-dimensional quasilinear utility function $U$ on $\mathscr{M}$ whose lexicographically ordered utility vectors preserve the ordering $\succ$. A set $\mathscr{M}$ is said to be a mixture space if, for all $0 \leq \lambda \leq 1$ and all ordered pair $(x, y) \in \mathscr{M} \times \mathscr{M}$, there is a unique element $x \lambda y$ in $\mathscr{M}$ such that, for all $x, y, z \in \mathscr{M}$ and all $\lambda, \mu \in[0,1]$,

M1. $x 1 y=x$,

M2. $x \lambda y=y(1-\lambda) x$,

M3. $(x \mu y) \lambda y=x(\lambda \mu) y$.

M4. $\quad(x \mu y) \lambda z=x(\lambda \mu)\left(y \frac{\lambda(1-\mu)}{1-\lambda \mu} z\right)$ for which $\lambda \mu \neq 1$.

An $n$-dimensional utility function $U=\left(u_{1}, \ldots, u_{n}\right)$ on $\mathscr{M}$ lexicographically preserves the ordering $\succ$ if, for all $x, y \in \mathscr{M}$,

$$
x \succ y \Longleftrightarrow U(x)>_{L} U(y),
$$

where $U(x)>_{L} U(y)$ means that $U(x) \neq U(y)$ and $u_{k}(x)>u_{k}(y)$ for the smallest $k$ for which $u_{k}(x) \neq u_{k}(y)$. We say that $U=\left(u_{1}, \ldots, u_{n}\right)$ is

\footnotetext{
*Institute of Policy and Planning Sciences, University of Tsukuba, 1-1-1 Tennoudai, Tsukuba, Ibaraki 305-8573, Japan; e-mail: nakamura@shako.sk.tsukuba.ac.jp
} 
quasilinear if, for each $k=1, \ldots, n$,

$$
u_{k}(x \lambda y)=\lambda u_{k}(x)+(1-\lambda) u_{k}(y)
$$

whenever $0 \leq \lambda \leq 1, u_{k}(x) \neq u_{k}(y)$, and $u_{j}(x)=u_{j}(y)$ for all $j<k$.

Hausner (1954) was the first to develop a lexicographic extension of the von Neumann and Morgenstern expected utility theory. His axioms yield an infinite-dimensional "linear" utility function $U$ on $\mathscr{M}$, whose lexicographically ordered infinite-dimensional utility vectors preserve $\succ$ on $\mathscr{M}$, where linearity of $U$ means that every component $u$ of $U$ is linear on $\mathscr{M}$, i.e., for all $x, y \in \mathscr{M}$ and all $0 \leq \lambda \leq 1, u(x \lambda y)=\lambda u(x)+(1-\lambda) u(y)$. Hausner noted that the dimension of $U$ is finite if the dimension of $\mathscr{M}$ is finite. Blume, Brandenburger, and Dekel (1991) used this fact to derive a lexicographic probability system for decision making under uncertainty. In general, however, Hausner's nonconstructive method says nothing about the size of dimensionality.

A hierarchical structure of finite-dimensionality was first axiomatically explored by Fishburn $(1971,1982)$ who presented a direct and constructive derivation of a finite-dimensional linear utility function $U$ on a mixture space $\mathscr{M}$. Several applications of this structure have recently appeared in a series of joint works by Fishburn and LaValle. For example, LaValle and Fishburn (1991, 1992) developed lexicographic extensions of subjective expected utility and derived the notion of matrix probabilities. Fishburn and LaValle (1992) examined decomposition structures of lexicographically ordered multiattribute expected utility.

Fishburn's hierarchical axiom is not preference-based so that it is not described directly by the preference order $\succ$ on $\mathscr{M}$. Recently, Nakamura (2000) developed such a preference-based hierarchical axiom which together with two independence axioms, also employed by Fishburn's hierarchical structure, is necessary and sufficient for the existence of a finite-dimensional linear utility function lexcicographically preserving $\succ$ on a mixture space $\mathscr{M}$. This paper generalizes Nakamura's result to the quasilinear case by introducing an axiom known as betweeness, dropping the independence axioms, and weakening his hierarchical axiom. We also show that the hierarchical axiom can be much simplified for the linear case.

The paper is organized as follows. Section 2 introduces and discuss a necessary and sufficeint axiom system for the existence of a finite-dimensional quasilinear utility function $U$ lexicographically preserving $\succ$, and presents the main theorem. Also, a simplified axiom system for the existence of a finite-dimensional linear utility function is presented. Then Section 3 provides the sufficiency proofs of the theorems. 


\section{Axioms and Theorems}

Let $\sim$ and $\succeq$ be defined in the ususal way: for all $x, y \in \mathscr{M}, x \sim y$ iff $\neg(x \succ y)$ and $\neg(y \succ x)$, and $x \succeq y$ iff $x \succ y$ or $x \sim y$. The relation $\succ$ is asymmetric if, for all $x, y \in \mathscr{M}, x \succ y$ implies $\neg(y \succ x)$, and negatively transitive if, for all $x, y \in \mathscr{M}, x \succ y$ implies $x \succ z$ or $z \succ y$. The binary relation $\succ$ is said to be a weak order if it is asymmetric and negatively transitive.

The following two independence axioms are necessary for lexicographic linear utility representations.

Independence Axiom I1. For all $x, y, z \in \mathscr{M}$ and all $0<\lambda<1$, if $x \succ y$, then $x \lambda z \succ y \lambda z$.

Independence Axiom 12. For all $x, y, z \in \mathscr{M}$ and all $0<\lambda<1$, if $x \sim y$, then $x \lambda z \sim y \lambda z$.

We say that axiom I1 (resp., I2) holds for a triple $(x, y, z)$ if $x \lambda z \succ y \lambda z$ (resp., $x \lambda z \sim y \lambda z)$ for all $0<\lambda \leq 1$. If $(\mathscr{M}, \succ)$ has a lexicographic linear utility representation, then axiom I1 (resp., I2) holds for every triple $(x, y, z)$ for which $x \succ y$ (resp., $x \sim y$ ).

It is well known that a weakly ordered $\succ$ on $\mathscr{M}$ satisfies the following Archimedean axiom together with axiom I1 if and only if dimension of a linear utility function $U$ that represents $\succ$ must be one, i.e., $U$ reduces to a unidimensional linear function on $\mathscr{M}$.

Archimedean Axiom AA. For all $x, y, z \in \mathscr{M}$, if $x \succ y$ and $y \succ z$, then $x \alpha z \succ y$ for some $0<\alpha<1$, and $y \succ x \beta z$ for some $0<\beta<1$.

We shall divide Archimedean assertions in axiom AA into two pieces for the later discussion as follows:

Upper Archimedean Axiom UAA. For all $x, y, z \in \mathscr{M}$, if $x \succ y$ and $y \succ z$, then $x \alpha z \succ y$ for some $0<\alpha<1$.

Lower Archimedean Axiom LAA. For all $x, y, z \in \mathscr{M}$, if $x \succ y$ and $y \succ z$, then $y \succ x \beta z$ for some $0<\beta<1$.

It will be shown in Lemma 5 of the next section that axioms UAA and LAA are equivalent if a weakly ordered $\succ$ on $\mathscr{M}$ satisfies the independence axiom I1. We say that axiom UAA (resp., LAA) holds for a triple $(x, y, z)$ if $x \succ y$, $y \succ z$, and $x \alpha z \succ y$ (resp., $y \succ x \alpha z$ ) for some $0<\alpha<1$.

In what follws, we shall introduce necessary and sufficient axioms for quasilinearity. To this end, we need to relax the Archimedean axiom AA and the independence axioms I1 and I2, since they are not necessary for quasilinearity. First we examine a simple example to see the relations among 
essentiality, defined below, of lexicographic quasilinear representation and axioms UAA, LAA, I1, and I2. Let $U=\left(u_{1}, \ldots, u_{n}\right)$ be a lexicographic quasilinear utility representation for $(\mathscr{M}, \succ)$. Given $x_{1}, \ldots, x_{m} \in \mathscr{M}$, a component function $u_{k}$ is said to be essential for an $m$-tuple $\left(x_{1}, \ldots, x_{m}\right)$ if $u_{i}\left(x_{1}\right)=\cdots=u_{i}\left(x_{m}\right)$ for all $i<k$, and $u_{k}(x) \neq u_{k}(y)$ for some $x, y \in$ $\left\{x_{1}, \ldots, x_{m}\right\}$.

Suppose that $x \succ y, y \succ z$, and $u_{k}$ is essential for $(x, y, z)$. Then it is seen to be necessary from the representation $U$ that $u_{k}$ is essential for the pair $(x, y)$ (resp., $(y, z)$ ) if and only if axiom UAA (resp., LAA) holds for $(x, y, z)$. It should be also observed that axiom I1 holds for $(x, y, z)$ (resp., $(y, z, x))$ if axiom UAA (resp., LAA) holds for $(x, y, z)$. It also follows as a necessary requirement that axiom I1 holds for triples $(x, y, x)$ and $(x, y, y)$ whenever there is a component function $u_{k}$ that is essential for the pair $(x, y)$. Furthermore, axiom I2 holds for triples $(x, y, x)$ and $(x, y, y)$ whenever there is no component function that is essential for the pair $(x, y)$.

To illustrate the above relations, we consider the following example.

Example 1 Suppose that $(\mathscr{M}, \succ)$ has a two-dimensional quasilinear representation $U=\left(u_{1}, u_{2}\right)$. Fix $x, y, z, w \in \mathscr{M}$, and define $u_{1}(x)=1$, $u_{1}(y)=u_{1}(z)=0, u_{1}(w)=-1, u_{2}(x)=1, u_{2}(y)=u_{2}(z)=u_{2}(w)=0$, and, for all $0<\lambda<1, u_{2}(x \lambda w)=-1, u_{2}(z \lambda w)=0$, and

$$
\begin{aligned}
& u_{2}(x \lambda y)= \begin{cases}1 & \text { if } \lambda \text { is rational, } \\
0 & \text { otherwise }\end{cases} \\
& u_{2}(x \lambda z)= \begin{cases}0 & \text { if } \lambda \text { is rational, } \\
1 & \text { otherwise }\end{cases}
\end{aligned}
$$

Since $y \sim z, x \frac{1}{2} y \succ x \frac{1}{2} z$, and $x \frac{1}{\sqrt{2}} z \succ x \frac{1}{\sqrt{2}} y$, axiom I1 failes to hold for $\left(x \frac{1}{2} y, x \frac{1}{2}, x\right)$, and axiom I2 fails to hold for $(y, z, x)$. Observe that $u_{1}$ is essential for $(y, z, x)$ and $\left(x \frac{1}{2} y, x \frac{1}{2} z, x\right)$, but not for $(y, z)$ and $\left(x \frac{1}{2} y, x \frac{1}{2} z\right)$. Since $u_{2}$ is essential for $\left(x \frac{1}{2} y, x \frac{1}{2} z\right)$ and $\left(x \frac{1}{2} y, x \frac{1}{2} z, x \frac{3}{4} w\right)$, axiom I1 holds for $\left(x \frac{1}{2} y, x \frac{1}{2} z, x \frac{3}{4} w\right)$. Axiom I1 also holds for $(z, w, x),(x, y, x)$, and $(x, y, y)$, since $u_{1}$ is essential for $(z, w, x),(z, w)$, and $(x, y)$. Essentiality of $u_{2}$ for $\left(y, x \frac{1}{2} w\right)$ implies axiom I1 holding for $\left(y, x \frac{1}{2} w, y\right)$ and $\left(y, x \frac{1}{2} w, x \frac{1}{2} w\right)$. Since $u_{1}$ and $u_{2}$ are not essential for $(y, z)$, axiom I2 holds for $(y, z, z)$ and $(y, z, y)$.

Now we state necessary and sufficient axioms as follows, which apply to all $x, y, z, x_{1}, \ldots, x_{n+1}, y_{1}, \ldots, y_{n+1} \in \mathscr{M}$, all $0<\lambda<1$, and all positive integers $n$.

Axiom A1. $\succ$ on $\mathscr{M}$ is a weak order.

Axiom A2. If $x \succ y$, then $x \succ x \lambda y \succ y$. 
Axiom A3. If $x \succ y$ and $y \succ z$, then

$$
\begin{aligned}
& x \alpha z \succ y \text { for some } 0<\alpha<1 \quad \Longrightarrow x \frac{1}{2} z \succ y \frac{1}{2} z ; \\
& y \succ x \beta z \text { for some } 0<\beta<1 \quad \Longrightarrow x \frac{1}{2} y \succ x \frac{1}{2} z .
\end{aligned}
$$

Axiom A4(n). If, for $i=1, \ldots, n$, either $x_{i}=x_{i+1}$ or $y_{i}=y_{i+1}$, and, for all $0<\lambda<1$,

$$
\begin{array}{ll}
x_{i} \succ y_{i} \quad \text { and } \quad x_{i} \lambda x_{i+1} \succ y_{i} \lambda y_{i+1} \quad \text { whenever } 1 \leq i \leq n \text { is odd, } \\
y_{i} \succ x_{i} \quad \text { and } \quad y_{i} \lambda y_{i+1} \succ x_{i} \lambda x_{i+1} \quad \text { whenever } 1 \leq i \leq n \text { is even, }
\end{array}
$$

then $\neg\left(y_{n+1} \succ x_{n+1}\right)$ if $n$ is odd, and $\neg\left(x_{n+1} \succ y_{n+1}\right)$ if $n$ is even.

A2 is known as the betweenness axiom, which requires that the independence axiom I1 holds for triples $(x, y, y)$ and $(x, y, x)$ for all $x, y \in \mathscr{M}$ such that $x \succ y$. A3 says that if either the upper or lower Archimedean axiom holds for a triple $(x, y, z)$, then a weak requirement of the independence axiom I1, i.e., $\lambda=\frac{1}{2}$, holds respectively for $(x, y, z)$ and $(y, z, x)$. It will be shown in Lamma 2 in the next section that axioms A1 and A2 force axiom I1 to fully hold for $(x, y, z)$ and $(y, z, x)$ in respective cases. $\mathrm{A} 4(n)$ is a relaxation of Archimedean axiom AA in which $n$ corresponds to a dimension of quasilinear representations. Nakamura (2000) adopted a stronger vesion of A4(n) in which it is dropped that, for $i=1, \ldots, n$, either $x_{i}=x_{i+1}$ or $y_{i}=y_{i+1}$. Assuming that A1 and A2 hold, A4(1) is equivalent to the Archimedean axiom AA.

The main theorem of the paper is stated as follows.

Theorem 1 Let $n$ be a positive integer. Axioms A1-A3, and A4(n) hold if and only if $(\mathscr{M}, \succ)$ has an at most $n$ dimensional lexicographic quasilinear representation.

A proof for sufficiency of the axioms will be deferred to the next section. Here we demonstrate neccesities of the axioms. Suppose that $(\mathscr{M}, \succ)$ has a lexicographic quasilinear representation $U=\left(u_{1}, \ldots, u_{n}\right)$.

It is easy to see that axiom A1 is necessary for the representation. For axiom A2, assume $x \succ y$, so that there is an integer $0 \leq k<n$ such that $u_{i}(x)=u_{i}(y)$ for $i=1, \ldots, k$ and $u_{k+1}(x)>u_{k+1}(y)$. By quasilinearity, $u_{i}(x)=u_{i}(x \lambda y)$ for $i=1, \ldots, k$ and $u_{k+1}(x)>u_{k+1}(x \lambda y)>u_{k+1}(y)$ for all $0<\lambda<1$. Hence $x \succ x \lambda y \succ y$.

Suppose that $x \succ y, z \succ w$, and either $x=z$ or $y=w$. Let $u_{k}$ and $u_{\ell}$ be respectively essential for the pairs $(x, y)$ and $(z, w)$. Then it follows from quasilinearity of $U$ that $k<\ell$ if and only if $x \lambda z \succ y \lambda w$ for all $0<\lambda<1$.

To show necessity of A3, assume that $x \succ y$ and $y \succ z$. Let $u_{k}$ and $u_{\ell}$ be respectively essential for the pairs $(x, y)$ and $(y, z)$. If $x \alpha z \succ y$ for some $0<\alpha<1$, then by the preceding paragraph, $k \leq \ell$. Since $u_{k}(y) \geq u_{k}(z)$ and $u_{k}(x)>u_{k}(y)$, it follows from quasilinearity that $u_{i}(x \lambda z)>u_{i}(y \lambda z)$ and 
$u_{k}(x \lambda z)>u_{k}(y \lambda z)$ for all $0<\lambda<1$ and $i=1, \ldots, k-1$. Hence $x \frac{1}{2} z \succ y \frac{1}{2} z$. When $y x \beta z$ for some $0<\beta<1$, it similarly follows that $x \frac{1}{2} y \succ x \frac{1}{2} z$.

To show necessity of A4(n), assume that the hypotheses of the axiom hold with $y_{n+1} \succ x_{n+1}$ if $n$ is odd, and $x_{n+1} \succ y_{n+1}$ if $n$ is even. For $i=1, \ldots, n+1$, let $u_{k_{i}}$ be essential for a pair $\left(x_{i}, y_{i}\right)$. Then $k_{i}<k_{i+1}$ for $i=1, \ldots, n$. However, $k_{n+1} \leq n$, a contradiction. Hence A4(n) holds for $U$.

Since the independence axiom I1 imlplies axioms A2 and A3, it follows from Theorem 1 that axioms A1, I1, and $\mathrm{A} 4(n)$ are also sufficient for the existence of an at most $n$ dimensional lexicographic quasilinear representation. Our proof of Theorem 1 shows Fishburn's hierarchical axiom with height $m$ for some $m \leq n$ holds. Fishburn (1982, Theorem 4 in Chapter 4) showed that axioms A1, I1, and I2 together with his hierarchical axiom with height $m$ is sufficient for the existence of an $m$ dimensional lexicographic linear representation. Therefore, It turns out that axioms A1, I1, I2, and A4(n) are necessary and sufficient for the existence of an at most $n$ dimensional lexicographic linear representation.

We show below that the extended Archimedean axiom A4(n) can be further simplified. To see this, consider a simple case. We assume that $(\mathscr{M}, \succ)$ has a two dimensional lexicographic quasiliear representation $U=$ $\left(u_{1}, u_{2}\right)$. Axiom A4(2) reads as follows: for all $x_{1}, x_{2}, x_{3}, y_{1}, y_{2}, y_{3} \in \mathscr{M}$,

Axioms A4(2) if either $x_{1}=x_{2}$ or $y_{1}=y_{2}$, either $x_{2}=x_{3}$ or $y_{2}=y_{3}$, $x_{1} \succ y_{1}, y_{2} \succ x_{2}, x_{1} \lambda x_{2} \succ y_{1} \lambda y_{2}$, and $y_{2} \lambda y_{3} \succ x_{2} \lambda x_{3}$ for all $0<\lambda<1$, then $\neg\left(x_{3} \succ y_{3}\right)$.

The requirements of the axioms can be divided into following two statements (i) and (ii).

(i) If $y \lambda z \succ x$ and $x \succ z \lambda w$ for all $0<\lambda<1$, then

$$
\begin{aligned}
& y \succ x \succ z \quad \Longrightarrow \quad \neg(w \succ x) ; \\
& z \succ x \succ w \quad \Longrightarrow \quad \neg(x \succ y) .
\end{aligned}
$$

(Note: leting $y_{1}=y_{2}=y_{3}=x, x_{1}=y, x_{2}=z$, and $x_{3}=w$ gives the former claim. The latter follows from letting $x_{1}=x_{2}=x_{3}=x, y_{1}=w, y_{2}=z$, and $y_{3}=y$.)

(ii) If $x \lambda z \succ y$ and $y \lambda w \succ z$ for all $0<\lambda<1$, then

$$
\begin{aligned}
& w \succ z \succ y \quad \Longrightarrow \quad \neg(y \succ x) ; \\
& x \succ y \succ z \quad \Longrightarrow \quad \neg(z \succ w) .
\end{aligned}
$$

(Note: the first claim obtains by letting $x_{1}=w, x_{2}=x_{3}=y, y_{1}=y_{2}=z$, and $y_{3}=x$. The second by letitng $x_{1}=x, x_{2}=x_{3}=z, y_{1}=y_{2}=y$, and $y_{3}=w$.) 
Assuming that axioms A1 and A2 hold, the two claims of (ii) can be respectively restated as follows:

(a) If $w \succ z \succ y \succ x$ and $y \lambda w \succ z$ for all $0<\lambda<1$, then $y \succ x \alpha z$ for some $0<\alpha<1$.

(b) If $x \succ y \succ z \succ w$ and $x \lambda z \succ y$ for all $0<\lambda<1$, then $z \succ$ yaw for some $0<\alpha<1$.

It will be clear from the proof of Thoerem 2 below that, assuming A1, I1, and I2, (b) implies (a) and (i). It may be regarded that (a) and (b) are respectively generalizations of the upper and lower Archimedean axioms. Thus $n$ dimensional version of (b) statement is described as axiom $\mathrm{A}^{*}(n)$ below, understood as applying to all $x_{1}, \ldots, x_{n+2} \in \mathscr{M}$ and all positive intergers $n$.

Axiom $\mathbf{A 4}^{*}(n)$. If $x_{1} \succ x_{2} \cdots \succ x_{n+2}$ and $x_{k} \lambda x_{k+2} \succ x_{k+1}$ for all $0<$ $\lambda<1$ and $k=1, \ldots, n-1$, then $x_{n+1} \succ x_{n} \alpha x_{n+2}$ for some $0<\alpha<1$.

Note that axiom $\mathrm{A}^{*}(1)$ is tantamount to the lower Archimedean axiom LAA.

The implication of $\mathrm{A} 4^{*}(n)$ is stated as follows.

Theorem 2 Let $n$ be a positive integer. Axioms A1, I1, I2, and $\mathrm{A}^{*}(n)$ hold if and only if $(\mathscr{M}, \succ)$ has an at most $n$ dimensional lexicographic linear representation.

A sufficiency proof will be deferred to the next section.

Before moving on to sufficiency proofs of the theorems, we show a simple example to illustrate a difference between quasilinear and linear representations. Let $X$ be a nondegenerate real interval with $\mathscr{P}$ the set of all gambles on $X$. A gamble is a simple probability distribution $P$ on $X$ such that $P(Y)=1$ for some finite subset $Y$ of $X$, and $\sum_{x \in X} P(x)=1$. A mixture of two gambles $P$ and $Q$ with respect to a number $0 \leq \lambda \leq 1$ is defined to be a comvex combination $P \lambda Q$ which is also a gamble yielding an outcome $x \in X$ with probability $\lambda P(x)+(1-\lambda) Q(x)$. Hence, $\mathscr{P}$ is a mixture space.

Let $E(f(x), P)=\sum_{x \in X} f(x) P(x)$ be the expected value of a real valued function $f$ on $X$ with respect to $P$. Let $\succ$ be a binary is preferred to relation on $\mathscr{P}$. Suppose that, for all $P, Q \in \mathscr{P}$,

$$
\begin{aligned}
P \succ Q \Longleftrightarrow\left(E(x, P),-\sum_{y \in X}(y-E(x, P))^{2}\right) \\
\\
>_{L}\left(E(x, Q), \sum_{y \in X}(y-E(x, Q))^{2}\right) .
\end{aligned}
$$

If elements in $X$ represent amounts of money considered as potential increments to present wealth, this representation of $\succ$ says that one gamble is 
preferred to a second if and only if the first has a larger expected monetary return, or the first has a smaller variance whenever the expected returns are equal. It is easy to see that $(\mathscr{P}, \succ)$ has a two-dimensional lexicographic linear representation $\left(x,-x^{2}\right)$, i.e., for all $P, Q \in \mathscr{P}$,

$$
P \succ Q \Longleftrightarrow\left(E(x, P),-E\left(x^{2}, P\right)\right)>_{L}\left(E(x, Q),-E\left(x^{2}, Q\right)\right) .
$$

There is no reason, however, why the dispersion of monetary returns for a gamble be measured by variances.

It might be appropriate to measure the dispersion by a more general measure which may also depend upon the expected values. Let $V$ be a real valued function on the 2-dimensional Euclidean space $\mathbb{R}^{2}$ for which $V(\tau, \mu)$ is nondecreasing in $\tau>0$ and nonincreasing in $\tau<0$, and $V(0, \mu)=0$ for all $\mu \in \mathbb{R}$. Then $\sum_{y \in X} V(y-E(x, P), E(x, P))$ may be regarded as a generalized variance of $P$, which may depend on mean values. Adopting $V$, a possible lexicographic quasilinear representation may read as follows: for all $P, Q \in \mathscr{P}$,

$$
\begin{aligned}
P \succ Q \Longleftrightarrow\left(E(x, P),-\sum_{y \in X} V(y-E(x, P), E(x, P))\right) \\
\\
>_{L}\left(E(x, Q),-\sum_{y \in X} V(y-E(x, Q), E(x, Q))\right) .
\end{aligned}
$$

A more specific measure for the generalized variance $V$ might be

$$
V(x-\mu, \mu)=|x-\mu|^{\sigma(\mu)},
$$

where the $\sigma(\mu)=2$ case reduces to the above lexicographic linear representation.

\section{Sufficiency Proofs}

Throughout the section we shall assume that $\mathscr{M}$ is a mixture space. We note that M1 through M3 imply that, for all $x, y \in \mathscr{M}$ and all $\alpha, \beta, \gamma \in[0,1]$,

M5. $(x \beta y) \alpha(x \gamma y)=x(\alpha \beta+(1-\alpha) \gamma) y$.

Sufficiency Proof of Theorem 1 Suppose that axioms A1-A3 and A $(n)$ for some integer $n>1$ hold. We shall prove the sufficiency of the axioms in three steps. In Step 1, we show that, for every nonempty closed preference interval, defined below, there is a linear function on it that weakly represents $\succ$ restricted to the interval. Then in Step 2, Fishburn's hierarchical axiom with height $n$ together with axioms A1-A3 implies the existence of $n$ dimensional lexicographic quasilinear representation. Finally, in Step 3, our weak Archimedean axiom A4 $(n)$ implies the hierarchical axiom with height $m \leq n$. 
Step 1. Following Fishburn (1982), we define a closed preference interval, denoted $\langle x, y\rangle$, by

$$
\langle x, y\rangle=\{z \in \mathscr{M}: x \succeq z \succeq y\} .
$$

It follows that $\langle x, y\rangle$ is empty if $y \succ x$, since $\succ$ is a weak order. By $\mathscr{N}$, we shall denote the set of all nonempty closed preference intervals with nonindifferent end points, i.e., $\mathscr{N}=\{\langle x, y\rangle: x \succ y\}$. When $\langle x, y\rangle,\langle z, w\rangle \in$ $\mathscr{N}$, the minimal element in $\mathscr{N}$ that includes both $\langle x, y\rangle$ and $\langle z, w\rangle$ is easily seen to be $\langle x, w\rangle \cup\langle z, y\rangle$.

The aim of the step is to prove the following claim.

Claim 1. Suppose that $\langle x, y\rangle \in \mathscr{N}$. Then there is a unique linear function $\phi_{x y}$ on $\langle x, y\rangle$ such that $\phi_{x y}(x)=1, \phi_{x y}(y)=0$, and, for all $z, w \in\langle x, r\rangle$, $z \succeq w \Longrightarrow \phi_{x y}(z) \geq \phi_{x y}(w)$.

The proof of the claim is deferred to the end of this step.

To prove the claim, we need the following three lemmas.

Lemma 1 (1) If $x \sim y$, then $x \sim x \lambda y$ for all $0<\lambda<1$.

(2) If $x \succ x \alpha y$ or $x \alpha y \succ y$ for some $0<\alpha<1$, then $x \succ y$.

(3) If $x \succ y$ and $x \succ z$, then $x \succ y \lambda z$ for all $0<\lambda<1$; if $y \succ x$ and $z \succ x$, then $y \lambda z \succ x$ for all $0<\lambda<1$.

Proof. (1) Suppose that $x \sim y$ and $x \alpha y \succ y$ for some $0<\alpha<1$. Then we shall derive a contradiction. When $y \succ x \alpha y$, a similar contradiction obtains. Hence by the definition of $\sim$, the desired result obtains.

By A1, A2, and M2, xay $\succ(x \alpha y) \beta y \succ y$ for some $0<\beta<1$, and $(x \alpha y) \beta y \succ x \gamma[(x \alpha y) \beta y]$ for all $0<\gamma<1$. We have

$$
\begin{aligned}
& x \gamma[(x \alpha y) \beta y]=x \gamma[x(\alpha \beta) y] \quad \text { (by M3) } \\
& =x \gamma[y(1-\alpha \beta) x] \quad \text { (by M2) } \\
& =[y(1-\alpha \beta) x](1-\gamma) x \quad \text { (by M2) } \\
& =y(1-\gamma)(1-\alpha \beta) x \quad(\text { by M3) } \\
& =x(\gamma+\alpha \beta-\alpha \beta \gamma) y \quad \text { (by M2). }
\end{aligned}
$$

Let $\gamma=\alpha(1-\beta) /(1-\beta \alpha)$, so $\gamma+\alpha \beta-\alpha \beta \gamma=\alpha$. Thus $x \gamma[(x \alpha y) \beta y]=x \alpha y$, a contradiction.

(2) This follows from (1) and A2.

(3) This follows from (2) and A1.

Lemma 2 (1) If $x \succ y$ and $y \succ z$, then

$$
\begin{gathered}
x \alpha z \succ y \text { for some } 0<\alpha<1 \quad \Longrightarrow \quad x \lambda z \succ y \lambda z \text { for all } 0<\lambda<1 ; \\
y \succ x \beta z \text { for some } 0<\beta<1 \Longrightarrow x \lambda y \succ x \lambda z \text { for all } 0<\lambda<1 .
\end{gathered}
$$


(2) If $x \succ y, y \succ z, x \alpha z \succ y, x \beta z \succ y$, and $0<\alpha<\beta<1$, then $x \lambda(x \beta z) \succ x \lambda y$ and $z \lambda(x \beta z) \succ z \lambda y$ for all $0<\lambda<1$.

(3) If $x \succ y, y \succ z, y \succ x \alpha z, y \succ x \beta z$, and $0<\alpha<\beta<1$, then $x \lambda y \succ x \lambda(x \alpha z)$ and $z \lambda y \succ z \lambda(x \alpha z)$ for all $0<\lambda<1$.

Proof. (1) Suppose that $x \succ y, y \succ z$, and $x \alpha z \succ y$ for some $0<\alpha<1$. When $y \succ x \beta z$ for some $0<\beta<1$, the proof is similar.

First we show that $x \lambda z \succ y \lambda z$ for all $\frac{1}{2} \leq \lambda<1$. Let $0 \leq \mu<1$. Note that, by A1 and A2, $x \succ y \succ y \mu z$, and

$$
\begin{aligned}
& x \frac{\alpha(1-\mu)}{1-\alpha \mu}(y \mu z)=x \frac{\alpha(1-\mu)}{1-\alpha \mu}(z(1-\mu) y) \quad(\text { by M2) } \\
& =(x \alpha z) \frac{1-\mu}{1-\alpha \mu} y \quad \text { (by M4) } \\
& \succ y \text {. }
\end{aligned}
$$

Then by A3, M2, and M3, $x \frac{1}{2}(y \mu z) \succ y \frac{1}{2}(y \mu z)=y \frac{1+\mu}{2} z$. It follows from Lemma $1(2)$ that $x \frac{1+\mu}{2} z \succ y \frac{1+\mu}{2} z$, since

$$
\begin{aligned}
& x \frac{1}{2}(y \mu z)=x \frac{1}{2}\left[\left(y \frac{1+\mu}{2} z\right) \frac{2 \mu}{1+\mu} z\right] \quad \text { (by M3) } \\
& =x \frac{1}{2}\left[z \frac{1-\mu}{1+\mu}\left(y \frac{1+\mu}{2} z\right)\right] \quad \text { (by M2) } \\
& =\left(x \frac{1+\mu}{2} z\right) \frac{1}{1+\mu}\left(y \frac{1+\mu}{2} z\right) \text { (by M4) } \\
& =\left(y \frac{1+\mu}{2} z\right) \frac{\mu}{1+\mu}\left(x \frac{1+\mu}{2} z\right) \text { (by M2) }
\end{aligned}
$$

Terefore, $\frac{1}{2} \leq \frac{1+\mu}{2}<1$, so that $x \lambda z \succ y \lambda z$ for all $\frac{1}{2} \leq \lambda<1$.

Since $x \succ y$ and $x \alpha z \succ y$, we have

$$
\begin{aligned}
x \lambda(x \alpha z) & =(z(1-\alpha) x)(1-\lambda) x & & (\text { by M2) } \\
& =z(1-\alpha)(1-\lambda) x & & (\text { by M3) } \\
& =x(\alpha+\lambda-\alpha \lambda) z . & & \text { (by M2) } \\
& \succ y & & \text { (by Lemma 1(3)) }
\end{aligned}
$$

Then by M3 and A3,

$$
\begin{aligned}
(x(\alpha+\lambda-\alpha \lambda) z) \frac{1}{2} z & =x \frac{\alpha+\lambda-\alpha \lambda}{2} z \\
& =\left(x \frac{1}{2} z\right)(\alpha+\lambda-\alpha \lambda) z \\
& \succ y \frac{1}{2} z .
\end{aligned}
$$

Since $x \frac{1}{2} z \succ y \frac{1}{2} z \succ z$, the analysis of the preceding paragraph applies to obtain that for all $\frac{1}{2} \leq \mu<1$,

$$
\left(x \frac{1}{2} z\right) \mu z \succ\left(y \frac{1}{2} z\right) \mu z .
$$

Letting $\mu=2 \lambda$, this is rearranged, by M3, to give $x \lambda z \succ y \lambda z$ for all $\frac{1}{2^{2}} \leq \lambda<$ $\frac{1}{2}$. This process continues indefinitely to conclude that, given any positive interger $n$,

$$
x \lambda z \succ y \lambda z \text { for all } \frac{1}{2^{n}} \leq \lambda<\frac{1}{2^{n-1}} .
$$


This completes the proof of (1).

(2) Suppose that $x \succ y, y \succ z, x \alpha z \succ y, x \beta z \succ y$, and $0<\alpha<\beta<1$. The latter claim in (2) follows from (1). Since, by A2, $x \succ x \beta z$, we are to show that $x \beta z \succ x \gamma y$ for some $0<\gamma<1$, so that the former claim follows again from (1).

Suppose on the contrary that there is no $0<\gamma<1$ such that $x \beta z \succ x \gamma y$. Thus it follows from A1 and A2 that $x \lambda y \succ x \beta z$ for all $0<\lambda<1$. Take any $0<\delta<\frac{\beta-\alpha}{1-\alpha}$. Then, by A2, $x \frac{\beta-\alpha}{1-\alpha} y \succ x \beta z \succ z$. We note that

$$
\begin{aligned}
\left(x \frac{\beta-\alpha}{1-\alpha} y\right) \frac{\beta(1-\alpha)(1-\delta)}{K} z & =\left(y \frac{1-\beta}{1-\alpha} x\right) \frac{\beta(1-\alpha)(1-\delta)}{K} z & & \text { (by M2) } \\
& =\left((y(1-\delta) x) \frac{1-\beta}{(1-\delta)(1-\alpha)} x\right) \frac{\beta(1-\alpha)(1-\delta)}{K} z & & \text { (by M3) } \\
& =(x \delta y) \frac{\beta(1-\beta)}{K}(x \beta z) & & \text { (by M2, M4) } \\
& \succ x \beta z, & & \text { (by A2) }
\end{aligned}
$$

where $K=(1-\alpha)(1-\delta)-(1-\beta)^{2}$. Therefore, by $(1),\left(x \frac{\beta-\alpha}{1-\alpha} y\right) \lambda z \succ(x \beta z) \lambda z$ for all $0<\lambda<1$. Let $\lambda=\alpha / \beta$, so we obtain

$$
\begin{aligned}
\left(x \frac{\beta-\alpha}{1-\alpha} y\right) \frac{\alpha}{\beta} z & =x \frac{\alpha(\beta-\alpha)}{\beta(1-\alpha)}\left(z \frac{(\beta-\alpha)(1-\alpha)}{\beta-2 \alpha \beta+\alpha^{2}} y\right) & & (\text { by M2, M4) } \\
& =(x \alpha z) \frac{\beta-\alpha}{\beta(1-\alpha)} y & & (\text { by M4) } \\
& \succ(x \beta z) \frac{\alpha}{\beta} z & & \\
& =x \alpha z . & & \text { (by M3) }
\end{aligned}
$$

Since $x \alpha z \succ y, \mathrm{~A} 2$ gives

$$
x \alpha z \succ(x \alpha z) \frac{\beta-\alpha}{\beta(1-\alpha)} y,
$$

a contradiction.

(3) This is similar to (2).

Lemma 3 (1) If $\langle x, y\rangle \in \mathscr{N}$ and $\lambda>\mu$, then $x \lambda y \succ x \mu y$.

(2) If $\langle x, y\rangle \in \mathscr{N}$ and $z \in\langle x, y\rangle$, then there is a unique $\lambda$ such that either

(a) $z \sim x \lambda y$, or

(b) $x \mu y \succ z$ for all $\mu \geq \lambda$; $z \succ x \mu y$ for all $\mu<\lambda$, or

(c) $\quad x \mu y \succ z$ for all $\mu>\lambda$; $z \succ x \mu y$ for all $\mu \leq \lambda$.

Proof. (1) Assume that $x \succ y$ and $\lambda>\mu$. Then $x \succ x \mu y$, by M1 and M2 if $\mu=0$, and by A2 if $\mu>0$. Hence $x \lambda y \succ x \mu y$, by M1 if $\lambda=1$, and by M2, M3, and A2 as follows if $\lambda<1: x \lambda y=y(1-\lambda) x=(y(1-\mu) x) \frac{1-\lambda}{1-\mu} x=$ $(x \mu y) \frac{1-\lambda}{1-\mu} x=x \frac{\lambda-\mu}{1-\mu}(x \mu y) \succ x \mu y$.

(2) Given $x \succeq z \succeq y$ and $x \succ y$, suppose first that $x \sim z$, so $z \sim x \succ y$. Then $z \sim x 1 y=x$ by M1, and $x 1 y \succ x \mu y$ for any $\mu<1$ by (1), so that $z \sim x \lambda y$ for a unique $\lambda$. A similar proof applies when $z \sim y$. Finally, 
suppose that $x \succ z \succ y$. It then follows from Lemma 1(3), M3, A1 that there is a unique $0 \leq \lambda \leq 1$ such that

$$
\begin{array}{ll}
x \alpha y \succ z & \text { for all } \alpha>\lambda, \\
z \succ x \beta y & \text { for all } \beta<\lambda .
\end{array}
$$

There are three possible cases: $z \sim x \lambda y$ for (a), $x \lambda y \succ z$ for (b), and $z \succ x \lambda y$ for (c).

Parts (b) and (c) in Lemma 3 reflect the absence of the Archimedean axiom AA. Note that in Fishburn (1982, Chapter 4), (1) and (2) in Lemma 3 are respectively named the conditions $\mathrm{J} 1$ and $\mathrm{J} 2 *$.

Proof of Claim 1. Suppose that $\langle x, y\rangle \in \mathscr{N}$. Define a real valued function $\phi_{x y}$ on $\langle x, y\rangle$ as follows: for every $z \in\langle x, y\rangle$, let $\phi_{x y}(z)=\lambda$ for a unique $\lambda$ whose existence is assured by Lemma 3(2). Then $\phi_{x y}(x)=1, \phi_{x y}(y)=0$, and, for all $z \in\langle x, y\rangle$,

$$
\begin{array}{ll}
x \lambda y \succ z & \text { for all } \lambda>\phi_{x y}(z), \\
z \succ x \lambda y & \text { for all } \lambda<\phi_{x y}(z) .
\end{array}
$$

Therefore, $z \succ w$ whenever $\phi_{x y}(z)>\phi_{x y}(w)$.

To show linearity of $\phi_{x y}$, let $z$ and $w$ be in $\langle x, y\rangle$ with $\alpha=\phi_{x y}(z)$ and $\beta=\phi_{x y}(w)$, and fix a $0<\lambda<1$ since linearity in $\lambda$ is obvious when $\lambda \in\{0,1\}$. We are to show that $\phi_{x y}(z \lambda w)=\lambda \phi_{x y}(z)+(1-\lambda) \phi_{x y}(w)$.

If $z \sim w$, then by the definition of $\phi_{x y}, \alpha=\beta$. Thus Lemma 1(1) gives $\phi_{x y}(z \lambda w)=\alpha$, so that $\phi_{x y}(z \lambda w)=\lambda \phi_{x y}(z)+(1-\lambda) \phi_{x y}(w)$. In the sequel, we shall assume that $z \succ w$. We have five cases to examine:

Case 1. $0<\beta \leq \alpha<1$,

Case 2. either $w=y$ and $0<\alpha<1$, or $z=x$ and $0<\beta<1$,

Case 3. either $w=y$ and $\alpha=1$, or $z=x$ and $\beta=0$,

Case 4. either $\beta=0$ and $0<\alpha$, or $\alpha=1$ and $\beta<1$,

Case 5. either $\alpha=\beta=0$ or $\alpha=\beta=1$.

Case 1. First we assume that $0<\beta<\alpha<1$. Let $\mu>\alpha$, so that $x \mu y \succ z$.

Suppose that $z \succ(x \mu y) \nu w$ for all $0<\nu<1$. Since $0<\beta$, it follows from Lemma 2(1) and M3 that $(x \mu y) \nu w \succ(x \mu y) \nu y$ for all $0<\nu<1$. Thus,

$$
\begin{aligned}
& z \succ(x \mu y) \nu y \quad \text { (by A1) } \\
& =x(\mu \nu) y \quad \text { (by M3) }
\end{aligned}
$$

Since $\mu>\alpha$, let $\nu$ be such that $\nu>\alpha / \mu$. Thus $z \succ x(\mu \nu) y$ for $\mu \nu>\alpha$, a contradiction. Hence we must have $(x \mu y) \gamma w \succ z$ for some $0<\gamma<1$. Then

By Lemma 2(1), $(x \mu y) \lambda w \succ z \lambda w$ for all $0<\lambda<1$. Let $\beta<\nu<\alpha$.

$$
x \nu y=(x \mu y) \frac{\nu}{\mu} y \succ w .
$$


Thus we have

$$
\begin{aligned}
(x \mu y) \lambda(x \nu y) & =x(\lambda \mu+(1-\lambda) \nu) y & & \text { (by M5) } \\
& \succ(x \mu y) \lambda w & & \text { (by Lemma 2(2)) } \\
& \succ z \lambda w & & \text { (by A1) }
\end{aligned}
$$

Since $\mu$ and $\nu$ are arbitrary as long as $\beta<\nu<\alpha<\mu$, we obtain that $x \gamma y \succ z \lambda w$ for all $\gamma>\lambda \alpha+(1-\lambda) \beta$.

It similarly follows from the preceding analysis that $z \lambda w \succ x \gamma y$ for all $\gamma<\lambda \alpha+(1-\lambda) \beta$. Hence, by definition, $\phi_{x y}(z \lambda w)=\lambda \alpha+(1-\lambda) \beta=$ $\lambda \phi_{x y}(z)+(1-\lambda) \phi_{x y}(w)$.

Next we assume that $0<\alpha=\beta<1$. By definition, we have

$$
\begin{array}{ll}
x \mu y \succ z \text { and } x \mu y \succ w & \text { for } \mu>\alpha, \\
z \succ x \mu y \text { and } w \succ x \mu y & \text { for } \mu<\alpha .
\end{array}
$$

By Lemma 1(3),

$$
\begin{array}{ll}
x \mu y \succ z \lambda w & \text { for } \mu>\alpha, \\
z \lambda w \succ x \mu y & \text { for } \mu<\alpha .
\end{array}
$$

Hence $\phi_{x y}(z \lambda w)=\alpha=\lambda \phi_{x y}(z)+(1-\lambda) \phi_{x y}(w)$.

Case 2. Assume that $w=y$ and $0<\alpha<1$. The proof for the other case is similar. By definition,

$$
\begin{array}{ll}
x \mu y \succ z & \text { for } \mu>\alpha, \\
z \succ x \mu y & \text { for } \mu<\alpha .
\end{array}
$$

By M3 and Lemma 2(1),

$$
\begin{array}{ll}
x(\lambda \mu) y=(x \mu y) \lambda y \succ z \lambda y & \text { for } \mu>\alpha, \\
z \lambda y \succ(x \mu y) \lambda y=x(\lambda \mu) y & \text { for } \mu<\alpha .
\end{array}
$$

Hence, $\phi_{x y}(z \lambda y)=\lambda \alpha=\lambda \phi_{x y}(z)+(1-\lambda) \phi_{x y}(y)$.

Case 3. Assume that $w=y$ and $\alpha=1$. The proof for the other case is similar. We have the following three subcases to examine.

Subcase $3.1 \quad \phi_{x y}(z \gamma y)=1$ for all $0<\gamma<1$,

Subcase $3.2 \quad \phi_{x y}(z \gamma y)=0$ for all $0<\gamma<1$,

Subcase $3.3 \quad 0<\phi_{x y}(z \gamma y)<1$ for some $0<\gamma<1$.

Subcase 3.1 Let $0<\gamma<1$. By A1 and A2, $x \succ z \gamma y \succ y$. Let $w^{*}=z \frac{1}{2} x$. We note that

$$
\begin{aligned}
& w^{*} \frac{2 \gamma}{1+\gamma} y=\left(z \frac{1}{2} x\right) \frac{2 \gamma}{1+\gamma} y \\
& =z \frac{\gamma}{1+\gamma}(x \gamma y) \quad \text { (by M4) } \\
& =z \frac{\gamma}{1+\gamma}(y(1-\gamma) x) \quad(\text { by M2) } \\
& =(z \gamma y) \frac{1}{1+\gamma} x \quad \text { (by M4) }
\end{aligned}
$$


Thus, by Lemma 1(3), A2, and M3, $w^{*} \mu y \succ z \gamma y$ for $\mu>2 \gamma /(1+\gamma)$. Since $\phi_{x y}(z \gamma y)=1, z \gamma y \succ x \mu y$ for all $0<\mu<1$. Thus, for all $0<\mu<1$,

$$
\begin{aligned}
z \gamma y & \succ(z \gamma y) \frac{\mu}{\mu+\gamma}(x \mu y) & & (\text { by A2) } \\
& =z \frac{\gamma \mu}{\mu+\gamma}\left(y \frac{\mu(1-\gamma)}{\mu+\gamma-\mu \gamma}(x \mu y)\right) & & \text { (by M4) } \\
& =z \frac{\gamma \mu}{\mu+\gamma}\left(x \frac{\gamma \mu}{\mu+\gamma-\mu \gamma} y\right) & & \text { (by M2, M3) } \\
& =\left(z \frac{1}{2} x\right) \frac{2 \gamma \mu}{\mu+\gamma} y & & \text { (by M4) } \\
& =w^{*} \frac{2 \gamma \mu}{\mu+\gamma} y, & &
\end{aligned}
$$

so that $z \gamma y \succ w^{*} \mu y$ for $\mu<2 \gamma /(1+\gamma)$. Hence, by definition, $\phi_{w^{*} y}(z \gamma y)=$ $2 \gamma /(1+\gamma)$.

Let $0<\delta<\gamma$. It follows from the preceding paragraph that $\phi_{w^{*} y}(z \delta y)=$ $2 \delta /(1+\delta)$. Since, by M3, $z \delta y=(z \gamma y) \frac{\delta}{\gamma} y$, Case 2 implies that

$$
\begin{aligned}
\phi_{w^{*} y}(z \delta y) & =\frac{\delta}{\gamma} \phi_{w^{*} y}(z \gamma y)+\left(1-\frac{\delta}{\gamma}\right) \phi_{w^{*} y}(y) \\
& =\frac{2 \delta}{1+\gamma},
\end{aligned}
$$

where $\phi_{w^{*} y}\left(w^{*}\right)=1$ and $\phi_{w^{*} y}(y)=0$. This is a contradiction. Hence this subcase cannot occur.

Subcase 3.2 Let $0<\gamma<1$ and $w^{*}=z \frac{1}{2} x$. By M2 and M4, $w^{*} \gamma y=$ $z \frac{\gamma}{2}\left(x \frac{\gamma}{2-\gamma} y\right)=x \frac{\gamma}{2}\left(z \frac{\gamma}{2-\gamma} y\right)$. By Lemma 1(3), A1, and A2, $x \succ w^{*} \gamma y \succ y$. By A2 and $\alpha=1, w^{*} \gamma y \succ x \frac{\gamma}{2-\gamma} y$.

For $\frac{\gamma}{2-\gamma} \leq \mu \leq 1$, we have

$$
\begin{array}{rlrl}
w^{*} \gamma y & =z \frac{\gamma}{2}\left(x \frac{\gamma}{2-\gamma} y\right) & \\
& =z \frac{\gamma}{2}\left((x \mu y) \frac{\gamma}{\mu(2-\gamma)} y\right) & & \text { (by M3) } \\
& =z \frac{\gamma}{2}\left(y \frac{2 \mu-\gamma \mu-\gamma}{\mu(2-\gamma)}(x \mu y)\right) & & \text { (by M2) } \\
& =\left(z \frac{\gamma \mu}{2 \mu-\gamma} y\right) \frac{2 \mu-\gamma}{2 \mu}(x \mu y) & & \text { (by M4) }
\end{array}
$$

We note that $f(\mu)=\gamma \mu /(2 \mu-\gamma)$ is strictly decreasing in $\mu$ for $\frac{\gamma}{2-\gamma} \leq \mu \leq 1$ with $f\left(\frac{\gamma}{2-\gamma}\right)=1$ and $f(1)=\frac{\gamma}{2-\gamma}$. By the hypothesis of the subcase, $x \mu y \succ$ $z f(\mu) y$ for $\frac{\gamma}{2-\gamma}<\mu \leq 1$, so by A2, $x \mu y \succ w^{*} \gamma y$. Therefore, by definition, $\phi_{x y}\left(w^{*} \gamma y\right)=\frac{\gamma}{2-\gamma}$.

Let $0<\delta<\gamma$. It follows from the preceding paragraph that $\phi_{x y}\left(w^{*} \delta y\right)=$ $\frac{\delta}{2-\delta}$. Since, by M3, $w^{*} \delta y=\left(w^{*} \gamma y\right) \frac{\delta}{\gamma} y$, Case 2 implies that

$$
\begin{aligned}
\phi_{x y}\left(w^{*} \delta y\right) & =\frac{\delta}{\gamma} \phi_{x y}\left(w^{*} \gamma y\right)+\left(1-\frac{\delta}{\gamma}\right) \phi_{x y}(y) \\
& =\frac{\delta}{2-\gamma} .
\end{aligned}
$$


This is a contradiction. Hence this subcase cannot occur.

Subcase 3.3 Fix a $0<\gamma<1$. Assume that $0<\phi_{x y}(z \gamma y)<1$. Note that $x \succ z \gamma y \succ y$. First we show that $\phi_{x y}(z \gamma y)=\gamma$, so $\phi_{x y}(z \gamma y)=$ $\gamma \phi_{x y}(z)+(1-\gamma) \phi_{x y}(y)$.

Assume first that $\phi_{x y}(z \gamma y)<\gamma$. By definition, $x \delta y \succ z \gamma y$ for all $\delta$ with $\phi_{x y}(z \gamma y)<\delta<\gamma$. Fix such a $\delta$. Then we have

$$
\begin{aligned}
x \frac{\delta}{\gamma} y & =y \frac{\gamma-\delta}{\gamma} x & & (\text { by M2) } \\
& =(y(1-\delta) x) \frac{\gamma-\delta}{\gamma(1-\delta)} x & & \text { (by M3) } \\
& =(x \delta y) \frac{\gamma-\delta}{\gamma(1-\delta)} x & & \text { (by M2) } \\
& =x \frac{\delta(1-\gamma)}{\gamma(1-\delta)}(x \delta y) & & \text { (by M2) } \\
& \succ x \frac{\delta(1-\gamma)}{\gamma(1-\delta)}(z \gamma y) & & \text { (by Lemma 2(2)) } \\
& =(z \gamma y) \frac{\gamma-\delta}{\gamma(1-\delta)} x & & \text { (by M2) } \\
& =z \frac{\gamma-\delta}{1-\delta}\left(y \frac{\gamma-\delta}{\gamma} x\right) & & \text { (by M4) } \\
& =z \frac{\gamma-\delta}{1-\delta}\left(x \frac{\delta}{\gamma} y\right) & & \text { (by M2) } \\
& \succ z & & \text { (by Lemma } 1(2))
\end{aligned}
$$

Since $\phi_{x y}(z)=1, z \succ x \frac{\delta}{\gamma} y$, a contradiction.

Assume next that $\phi_{x y}(z \gamma y)>\gamma$. By definition, $z \gamma y \succ x \delta y$ for all $\delta$ with $\gamma \leq \delta<\phi_{x y}(z \gamma y)$. Fix such a $\delta$ with $\delta \neq \gamma$. Let $\gamma<\mu<\delta$. Since $z \succ x \delta y \succ y$ and $z \gamma y \succ x \delta y$, we have

$$
\begin{aligned}
z \mu y & =z \frac{\mu-\gamma}{1-\gamma}(z \gamma y) & & (\text { by M2, M3) } \\
& \succ x \delta y . & & \text { (by Lemma 1(3)) }
\end{aligned}
$$

It follows from Lemma 2(2) that $z \nu(z \mu y) \succ z \nu(x \delta y)$ for all $0<\nu<1$. Let $\nu=\mu(1-\delta) /(\delta(1-\mu))$, so that

$$
\begin{aligned}
z \frac{\mu}{\delta} y & =z \frac{\mu(1-\delta)}{\delta(1-\mu)}(z \mu y) \quad(\text { by M2, M3) } \\
& \succ z \frac{\mu(1-\delta)}{\delta(1-\mu)}(x \delta y) \\
& =x \frac{\delta-\mu}{1-\mu}\left(z \frac{\mu}{\delta} y\right) . \quad(\text { by M2, M4) }
\end{aligned}
$$

By Lemma 1(2), $z \frac{\mu}{\delta} y \succ x$. On the other hand, Lemma 1(3) gives $x \succ z \frac{\mu}{\delta} y$, since $x \succ z$ and $x \succ y$. This is a contradiction. Hence we must have $\phi_{x y}(z \gamma y)=\gamma$.

Next we show that $\phi_{x y}(z \delta y)=\delta$ for all $\delta \neq \gamma$. Since, by M3, $z \delta y=$ $(z \gamma y) \frac{\delta}{\gamma} y$ for $0<\delta<\gamma$, Case 2 gives

$$
\phi_{x y}(z \delta y)=\frac{\delta}{\gamma} \phi_{x y}(z \gamma y)=\delta .
$$

Assume $\gamma<\delta<1$. Take any $\mu$ for which $\gamma<\mu<1$. We have

$$
\begin{aligned}
(x \mu y) \frac{\delta-\gamma}{\delta-\mu \gamma}(z \delta y) & =x \frac{\mu(\delta-\gamma)}{\delta-\mu \gamma}\left(y \frac{\delta-\gamma}{\delta}(z \delta y)\right) & & \text { (by M4) } \\
& =x \frac{\mu(\delta-\gamma)}{\delta-\mu \gamma}(z \gamma y) & & \text { (by M2, M3) }
\end{aligned}
$$


If $\delta<\mu$ and $z \delta y \succ x \mu y$, then by A2, $x \frac{\mu(\delta-\gamma)}{\delta-\mu \gamma}(z \gamma y) \succ x \mu y$. Thus, by Case 2 ,

$$
\begin{aligned}
\phi_{x y} & \left(x \frac{\mu(\delta-\gamma)}{\delta-\mu \gamma}(z \gamma y)\right) \\
= & \frac{\mu(\delta-\gamma)}{\delta-\mu \gamma} \phi_{x y}(x)+\frac{\delta(1-\mu)}{\delta-\mu \gamma} \phi_{x y}(z \gamma y) \\
= & \frac{\mu(\delta-\gamma)+\delta \gamma(1-\mu)}{\delta-\mu \gamma},
\end{aligned}
$$

which is strictly smaller than $\mu$, a contradiction.

If $\mu<\delta$ and $x \mu y \succ z \delta y$, then it follows that

$$
\phi_{x y}\left(x \frac{\mu(\delta-\gamma)}{\delta-\mu \gamma}(z \gamma y)\right)>\mu,
$$

a contradiction. Hence we must have that

$$
\begin{array}{ll}
x \mu y \succ z \delta y \quad \text { for } \mu>\delta, \\
z \delta y \succ x \mu y \quad \text { for } \mu<\delta,
\end{array}
$$

which give $\phi_{x y}(z \delta y)=\delta$.

Case 4. Assume that $\beta=0$ and $\alpha>0$. Since $w=y$ is covered by Case 3, we assume that $w \neq y$. The proof for the other case is similar.

Let $0<\gamma<\alpha$. It follows from Cases 2 and 3 that $\phi_{x y}\left(z \frac{\gamma}{\alpha} y\right)=\gamma$ and $\phi_{x y}(x \gamma w)=\gamma$. By Case 1, for all $0<\mu<1, \phi_{x y}\left(\left(z \frac{\gamma}{\alpha} y\right) \mu(x \gamma w)\right)=\gamma$. We note

$$
\begin{aligned}
\left(z \frac{\gamma}{\alpha} y\right) \frac{\alpha(1-\gamma)}{2 \alpha-\gamma(1+\alpha)}(x \gamma w) & =z \frac{\gamma(1-\gamma)}{2 \alpha-\gamma(1+\alpha)}\left(y \frac{1-\gamma}{2-\gamma}(x \gamma w)\right) \\
& =z \frac{\gamma(1-\gamma)}{2 \alpha-\gamma(1+\alpha)}\left((w(1-\gamma) x) \frac{1}{2-\gamma} y\right) \\
& =z \frac{\gamma(1-\gamma)}{2 \alpha-\gamma(1+\alpha)}\left(w \frac{1-\gamma}{2-\gamma}(x \gamma y)\right) \\
& =\left(z \frac{\gamma}{\alpha} w\right) \frac{\alpha(1-\gamma)}{2 \alpha-\gamma(1+\alpha)}(x \gamma y)
\end{aligned}
$$

Then, applying Case 2, it follows from the analysis similar to the last paragraph of Subcase 3.3 that $\phi_{x y}\left(z \frac{\gamma}{\alpha} w\right)=\gamma$. Since $\gamma$ is arbitrary as long as $0<\gamma<\alpha$, we obtain that $\phi_{x y}(z \lambda w)=\lambda \phi_{x y}(z)+(1-\lambda) \phi_{x y}(w)$.

Case 5. Similar to the proof for Case 1 when $\alpha=\beta$.

Step 2. We introduce Fishburn's hierarchical axiom with height $n$, named $\mathrm{H}(n)$, and prove the following claim.

Claim 2 Let $n$ be a positive integer. Suppose that $\mathscr{M}$ is a mixture space that satisfies axioms $\mathrm{A} 1-\mathrm{A} 3$, and $\mathrm{H}(n)$. Then $(\mathscr{M}, \succ)$ has an $n$-dimensional lexicographic quasilinear representation. 
To state $\mathrm{H}(n)$, we need several notations and definitions. We define two binary relations, $\sqsupseteq$ and $\sqsupset^{*}$, on $\mathcal{N}$ by

$$
\begin{aligned}
\langle x, y\rangle \sqsupseteq\langle z, w\rangle & \Longleftrightarrow\langle x, y\rangle \supseteq\langle z, w\rangle \text { and } \phi_{x y}(z)>\phi_{x y}(w), \\
\langle x, y\rangle \sqsupset^{*}\langle z, w\rangle & \Longleftrightarrow\langle x, y\rangle \supseteq\langle z, w\rangle \text { and } \phi_{x y}(z)=\phi_{x y}(w) .
\end{aligned}
$$

When $\langle x, y\rangle \supseteq\langle z, w\rangle$ and both intervals are in $\mathcal{N}$, exactly one of $\langle x, y\rangle \sqsupseteq$ $\langle z, w\rangle$ and $\langle x, y\rangle \sqsupset^{*}\langle z, w\rangle$ must hold. It is easy to see from the definitions that $\sqsupseteq$ is reflexive and $\sqsupset^{*}$ is irreflexive. The failure of $\phi_{x y}(z)=\phi_{x y}(w)$ can happen only if the Archimedean axiom AA is false.

We now define a key binary relation $=_{0}$ on $\mathcal{N}$ induced by $\sqsupseteq$ as follows:

$$
\begin{aligned}
\langle x, y\rangle={ }_{0}\langle z, w\rangle \quad \text { iff } & \langle x, w\rangle \cup\langle z, y\rangle \sqsupseteq\langle x, y\rangle \\
& \text { and }\langle x, w\rangle \cup\langle z, y\rangle \sqsupseteq\langle z, w\rangle .
\end{aligned}
$$

Assuming that axioms A1, I1, and I2 hold, Fishburn (1982, Chapter 4) proved that

(a) $\sqsupseteq$ on $\mathcal{N}$ is reflexive and transitive,

(b) $=_{0}$ on $\mathcal{N}$ is an equivalence relation.

Thus we can partition $\mathscr{N}$ into equivalence classes by $=_{0}$, and let $\mathscr{N}_{0}=$ $\mathscr{N} /=_{0} . \mathscr{N}_{0}$ consists of a single class $\mathscr{N}$ if and only if the Archimedean axiom AA holds. Given an equivalence class $A \in \mathscr{N}_{0}$, let $\mathscr{M}(A)$ denote the set of all elements in $\mathscr{M}$ that appear in at least one interval in $A$, i.e., $\mathscr{M}(A)=\cup_{A}\langle x, y\rangle$. Then Fishburn (1982, Chapter 4) also showed that axioms A1, I1, and I2 imply that

(c) each $\mathscr{M}(A)$ for $A \in \mathscr{N}_{0}$ is a mixture set,

(d) for any two distinct $A, B \in \mathscr{N}_{0}$, either $\mathscr{M}(A) \cap \mathscr{M}(B)=\emptyset$ or $\mathscr{M}(A) \supset$ $\mathscr{M}(B)$ or $\mathscr{M}(B) \supset \mathscr{M}(A)$.

Now we are ready to state Fishburn's hierarchical axiom. Adjacent mixture subsets induced by $=_{0}$ are identified by $\supset_{1}$, so that, for all $A, B \in \mathscr{N}_{0}$,

$$
\begin{aligned}
& \mathscr{M}(A) \supset_{1} \mathscr{M}(B) \\
& \quad \text { iff } \mathscr{M}(A) \supset \mathscr{M}(B) \text { and } \mathscr{M}(A) \supset \mathscr{M}(C) \supset \mathscr{M}(B) \text { for no } C \in \mathscr{N}_{0} .
\end{aligned}
$$

Furthermore, mixture subsets separated by $k-1$ other ordered mixture subsets are identified by $\supset_{k}$, so that, for $k \geq 2$ and for all $A, B \in \mathscr{N}_{0}$,

$$
\begin{aligned}
& \mathscr{M}(A) \supset_{k} \mathscr{M}(B) \\
& \quad \text { iff } \mathscr{M}(A) \supset_{1} \mathscr{M}(C) \text { and } \mathscr{M}(C) \supset_{k-1} \mathscr{M}(B) \text { for some } C \in \mathscr{N}_{0} .
\end{aligned}
$$

Given a positive integer $n$, Fishburn's hierarchical axiom with height $n$ is described as follows. 
$\mathbf{H}(n)$. For $n=1$, there is no $A, B \in \mathscr{N}_{0}$ such that $\mathscr{M}(A) \supset \mathscr{M}(B)$. For $n>1$, there are some $A, B \in \mathscr{N}_{0}$ such that $\mathscr{M}(A) \supset_{n-1} \mathscr{M}(B)$, and for all $A, B \in \mathscr{N}_{0}$, if $\mathscr{M}(A) \supset \mathscr{M}(B)$ then $\mathscr{M}(A) \supset_{k} \mathscr{M}(B)$ for some $1 \leq k \leq n-1$.

Proof of Claim 2. Let $n$ be a positive integer. Assume that axioms A1$\mathrm{A} 3$, and $\mathrm{H}(n)$ hold for $(\mathscr{M}, \succ)$. Fishburn (1982, Theorem 4.3) proved the lexicographic quasilinear representation by assuming axioms A1, I1, I2, and $\mathrm{H}(n)$ for $(\mathscr{M}, \succ)$. He first derived the facts (a)-(d), and then came to the representation using (a)-(d) and a partial weak representation as in Claim 1 of Step 1. Since his proofs for (a)-(d) are valid under our assumption that axioms A1-A3 hold, his derivation of the representaiton also applies to obtain the desired result.

Step 3. The sufficiency proof is completed by showing that axiom $\mathrm{H}(m)$ holds for some $m \leq n$. Suppose on the contrary that there are $A_{1}, \ldots A_{n+1} \in$ $\mathscr{N}_{0}$ such that $\mathscr{M}\left(A_{1}\right) \supset \cdots \supset \mathscr{M}\left(A_{n+1}\right)$. For every $i=1, \ldots, n+1$, we take a nonempty interval $\left\langle x_{i}, y_{i}\right\rangle \in A_{i}$.

Now we shall construct a decreasing sequence, $\left\langle z_{1}, w_{1}\right\rangle, \ldots,\left\langle z_{n+1}, w_{n+1}\right\rangle$, backwardly as follows. Let $\left\langle z_{n+1}, w_{n+1}\right\rangle=\left\langle x_{n+1}, y_{n+1}\right\rangle$. Then for $1 \leq k \leq n$, let

$$
\left\langle z_{k}, w_{k}\right\rangle=\left\langle z_{k+1}, y_{k}\right\rangle \cup\left\langle x_{k}, w_{k+1}\right\rangle .
$$

Thus by construction, $\left\langle z_{1}, w_{1}\right\rangle \supseteq \cdots \supseteq\left\langle z_{n+1}, w_{n+1}\right\rangle$.

The following lemma is proved by Nakamura (2000, Lemma 7), assuming axioms A1, I1, and I2. However, his proof is also valid under our assumption.

Lemma 4 If $\langle x, y\rangle \in A,\langle z, w\rangle \in B$, and $\mathscr{M}(A) \supset \mathscr{M}(B)$, then $\langle x, w\rangle \cup$ $\langle z, y\rangle \sqsupseteq\langle x, y\rangle$ and $\langle x, w\rangle \cup\langle z, y\rangle \sqsupset^{*}\langle z, w\rangle$

It follows from Lemma 4 that $\left\langle z_{k}, w_{k}\right\rangle \sqsupseteq\left\langle x_{k}, y_{k}\right\rangle$ and $\left\langle z_{k}, w_{k}\right\rangle \sqsupset^{*}\left\langle z_{k+1}, w_{k+1}\right\rangle$. Therefore, for $k=1, \ldots, n,\left\langle z_{k}, w_{k}\right\rangle={ }_{0}\left\langle x_{k}, y_{k}\right\rangle$, and

$$
\left\langle z_{1}, w_{1}\right\rangle \sqsupset^{*} \cdots \sqsupset^{*}\left\langle z_{n+1}, w_{n+1}\right\rangle .
$$

For every $k=1, \ldots, n$, we take an element $x_{k}^{*} \in\left\langle z_{k}, w_{k}\right\rangle$ for which

$$
\phi_{z_{k} w_{k}}\left(x_{k}^{*}\right) \neq \phi_{z_{k} w_{k}}\left(z_{k+1}\right) \text {. }
$$

To show a violation of $\mathrm{A}(n)$, let a sequence of pairs of elements in $\mathscr{M}$, $\left(x_{1}^{\prime}, y_{1}^{\prime}\right), \ldots,\left(x_{n+1}^{\prime}, y_{n+1}^{\prime}\right)$, be backwardly defined as follows. Take any $x_{n+1}^{\prime}$ and $y_{n+1}^{\prime}$ in $\left\langle z_{n+1}, w_{n+1}\right\rangle$ to satisfy that $x_{n+1}^{\prime} \succ y_{n+1}^{\prime}$ if $n+1$ is odd, and $y_{n+1}^{\prime} \succ x_{n+1}^{\prime}$ if $n+1$ is even. Then, for $k=n, n-1, \ldots, 1$, we define $x_{k}^{\prime}$ and $y_{k}^{\prime}$ by

$$
z_{k+1} \succ x_{k}^{*} \Longrightarrow \begin{cases}x_{k}^{\prime} & = \begin{cases}x_{k}^{*} & \text { if } k \text { is even, } \\ x_{k+1}^{\prime} & \text { if } k \text { is odd },\end{cases} \\ y_{k}^{\prime}= \begin{cases}y_{k+1}^{\prime} & \text { if } k \text { is even, } \\ x_{k}^{*} & \text { if } k \text { is odd },\end{cases} \end{cases}
$$




$$
x_{k}^{*} \succ z_{k+1} \Longrightarrow \begin{cases}x_{k}^{\prime} & = \begin{cases}x_{k}^{*} & \text { if } k \text { is odd }, \\ x_{k+1}^{\prime} & \text { if } k \text { is even },\end{cases} \\ y_{k}^{\prime}= \begin{cases}y_{k+1}^{\prime} & \text { if } k \text { is odd }, \\ x_{k}^{*} & \text { if } k \text { is even } .\end{cases} \end{cases}
$$

It is easy to see by construction that $x_{1}^{\prime} \succ y_{1}^{\prime}$ and, for $k=1, \ldots, n$,

$$
x_{k}^{\prime} \succ y_{k}^{\prime} \Longleftrightarrow y_{k+1}^{\prime} \succ x_{k+1}^{\prime},
$$

and either $x_{k}^{\prime}=x_{k+1}^{\prime}$ or $y_{k}^{\prime}=y_{k+1}^{\prime}$. Also we have that, for $k=1, \ldots, n$,

$$
\begin{array}{ll}
\left\langle x_{k}^{\prime}, y_{k}^{\prime}\right\rangle \sqsupset^{*}\left\langle y_{k+1}^{\prime}, x_{k+1}^{\prime}\right\rangle & \text { if } k \text { is odd, } \\
\left\langle y_{k}^{\prime}, x_{k}^{\prime}\right\rangle \sqsupset^{*}\left\langle x_{k+1}^{\prime}, y_{k+1}^{\prime}\right\rangle & \text { if } k \text { is even. }
\end{array}
$$

Therefore, for $k=1, \ldots, n$,

$$
\begin{aligned}
& x_{k}^{\prime} \lambda x_{k+1}^{\prime} \succ y_{k}^{\prime} \lambda y_{k+1}^{\prime} \text { for all } 0<\lambda \leq 1 \text { if } k \text { is odd, } \\
& y_{k}^{\prime} \lambda y_{k+1}^{\prime} \succ x_{k}^{\prime} \lambda x_{k+1}^{\prime} \text { for all } 0<\lambda \leq 1 \text { if } k \text { is even, }
\end{aligned}
$$

which clearly violate $\mathrm{A}(n)$. This completes the sufficiency proof of Theorem 1.

Sufficiency Proof of Theorem 2 Suppose that axioms A1, I1, I2, and $\mathrm{A} 4^{*}(n)$ hold. When $n=1$, it suffices to show the following lemma.

Lemma 5 The lower Archimedean axiom LAA holds if and only if the upper Archimedean axiom UAA holds.

Proof. Suppose that the lower Archimedean axiom LAA holds, i.e., for $x, y, z \in \mathscr{M}, x \succ y \succ z$ and $y \succ x \alpha z$ for some $0<\alpha<1$. We are to show that $x \beta z \succ y$ for some $0<\beta<1$. When the upper Archimedean axiom UAA holds, it similarly follows that axiom LAA holds.

First we assume that $y \succ x \lambda z$ for all $0<\lambda<1$. Let $0<\gamma<1$. By I1, $x \gamma y \succ x \gamma z \succ y \gamma z$. Take any $\lambda$ for which $\gamma<\lambda<1$. Since $x \gamma z=(x \lambda z) \frac{\gamma}{\lambda} z$ by M3, and $y \succ x \lambda z$, I1 gives $y \frac{\gamma}{\lambda} z \succ x \gamma z$. Let $f(\lambda)=\gamma(1-\lambda) /(\lambda+\gamma-2 \lambda \gamma)$. We note that

$$
\begin{aligned}
(x \gamma z) f(\lambda)\left(y \frac{\gamma}{\lambda} z\right) & =x(\gamma f(\lambda))\left(z \frac{f(\lambda)(1-\gamma)}{1-\gamma f(\lambda)}\left(y \frac{\gamma}{\lambda} z\right)\right) \\
& =x(\gamma f(\lambda))\left(y \frac{\gamma(1-f(\lambda))}{\lambda(1-\gamma f(\lambda))} z\right) \\
& =x(\gamma f(\lambda))\left(z \frac{\lambda-\gamma+\gamma f(\lambda)(1-\lambda)}{\lambda(1-\gamma f(\lambda))} y\right) \\
& =x(\gamma f(\lambda))\left((y \gamma z) \frac{\lambda-\gamma+\gamma f(\lambda)(1-\lambda)}{\lambda(1-\gamma)(1-\gamma f(\lambda))} y\right) \\
& =x(\gamma f(\lambda))\left(y \frac{\gamma(1-f(\lambda))-\lambda \gamma(1-\gamma f(\lambda))}{\lambda(1-\gamma)(1-\gamma f(\lambda))}(y \gamma z)\right) \\
& =x(\gamma f(\lambda))\left(y \frac{f(\lambda)(1-\gamma)}{1-\gamma f(\lambda)}(y \gamma z)\right) \\
& =(x \gamma y) f(\lambda)(y \gamma z)
\end{aligned}
$$


Thus by I1, $(x \gamma y) f(\lambda)(y \gamma z) \succ x \gamma z$. Since $f(1)=0, f(\gamma)=\frac{1}{2}$, and $f$ is strictly decreasing in $\lambda$ for which $\gamma \leq \lambda \leq 1, \mathrm{~A} 4^{*}(1)$ is violated. Hence $x \beta z \succeq y$ for some $0<\beta<1$.

If $x \beta z \sim y$ for some $0<\beta<1$, then by A1 and I1, $x \gamma(x \beta z) \succ y$, so by M2 and M3, $x(\beta+\gamma-\beta \gamma) z \succ y$.

Suppose that $n>1$. Since axioms A2 and A3 hold, Claim 2 in the sufficiency proof of Theorem 1 obtains if axiom $\mathrm{H}(n)$ also holds. It then follows from Theorem 4 in Fishburn (1982, Chapter 4) that the lexicographic representation must be linear. It remains to show that axiom $\mathrm{H}(m)$ holds for some $m \leq n$. Suppose on the contrary that $\mathrm{H}(m)$ fails to hold for any $m \leq n$. As shown in Step 3 of the sufficiency proof of Theorem 1, there is a decreasing sequence of preference intervals, $\left\langle z_{1}, w_{1}\right\rangle, \ldots,\left\langle z_{n+1}, w_{n+1}\right\rangle$, such that

$$
\left\langle z_{1}, w_{1}\right\rangle \sqsupset^{*} \cdots \sqsupset^{*}\left\langle z_{n+1}, w_{n+1}\right\rangle .
$$

Note that $\phi_{z_{n+1} w_{n+1}}\left(w_{n+1}\right)=0, \phi_{z_{n+1} w_{n+1}}\left(z_{n+1}\right)=1$ and $0=\phi_{z_{k} w_{k}}\left(w_{k}\right) \leq$ $\phi_{z_{k} w_{k}}\left(z_{k+1}\right)=\phi_{z_{k} w_{k}}\left(w_{k+1}\right) \leq \phi_{z_{k} w_{k}}\left(z_{k}\right)=1$ for $k=1, \ldots, n$. Let $K_{0}=\{k:$ $\phi_{z_{k} w_{k}}\left(z_{k+1}\right)=1$ and $\left.1 \leq k \leq n\right\}$. We have two cases to examine: $K_{0}=\emptyset$; $K_{0} \neq \emptyset$.

Case $1\left(K_{0}=\emptyset\right)$ For $k=1, \ldots, n$, take any $x_{k}^{*} \in\left\langle z_{k}, w_{k}\right\rangle$ such that $\phi_{z_{k} w_{k}}\left(x_{k}^{*}\right)>\phi_{z_{k} w_{k}}\left(z_{k+1}\right)$. Then $x_{1}^{*} \succ x_{2}^{*} \succ \cdots \succ x_{n}^{*} \succ z_{n+1} \succ w_{n+1}$, and

$$
x_{k}^{*} \lambda x_{k+2}^{*} \succ x_{k+1}^{*} \text { for } k=1, \ldots, n,
$$

where $x_{n+1}^{*}=z_{n+1}$ and $x_{n+2}^{*}=w_{n+1}$. This contradicts $\mathrm{A} 4^{*}(n)$.

Case $2\left(K_{0} \neq \emptyset\right)$ Let $k_{0}=\min K_{0}$. Let $0<\lambda<1$. For every $\ell=$ $k_{0}+1, \ldots, n+1$, let $z_{\ell}^{1}=w_{k_{0}} \lambda z_{\ell}$ and $w_{\ell}^{1}=w_{k_{0}} \lambda w_{\ell}$. Then $\left\langle z_{1}^{1}, w_{1}^{1}\right\rangle \sqsupset^{*}$ $\cdots \sqsupset^{*}\left\langle z_{n+1}^{1}, w_{n+1}^{1}\right\rangle$, where $z_{i}^{1}=z_{i}$ and $w_{i}^{1}=w_{i}$ for $i=1, \ldots, k_{0}$. Let $K_{1}=\left\{k: \phi_{z_{k}^{1} w_{k}^{1}}\left(z_{k+1}^{1}\right)=1\right.$ and $\left.1 \leq k \leq n\right\}$. If $K_{1}=\emptyset$, then Case 1 applies to obtain a contradiction. Thus assume that $K_{1} \neq \emptyset$. By construction, $k_{0}<\min K_{1}$.

A similar modification of $z_{1}^{1}, \ldots, z_{n+1}^{1}, w_{1}^{1}, \ldots, w_{n+1}^{1}$ gives a decreasing sequence, $\left\langle z_{1}^{2}, w_{1}^{2}\right\rangle \sqsupset^{*} \ldots \sqsupset^{*}\left\langle z_{n+1}^{2}, w_{n+1}^{2}\right\rangle$ with $K_{2}=\left\{k: \phi_{z_{k}^{2} w_{k}^{2}}\left(z_{k+1}^{2}\right)=\right.$ 1 and $1 \leq k \leq n\}$. If $K_{2}=\emptyset$, then Case 1 again gives a contradiction. If $K_{2} \neq \emptyset$, then $\min K_{1}<\min K_{2}$. This modification process continues up to at most $n$ times, and gives a contradiction.

This completes the sufficiency proof of Theorem 2 .

\section{References}

Blume, L., Brundenburger, A. and Dekel, E. (1991) Lexicographic probabilities and choice under uncertainty. Econometrica 59, 61-79. 
Fishburn, P.C. (1971) A study of lexicographic expected utility. Management Sciences 17, 672-678.

Fishburn, P.C. (1982) The Foundations of Expected Utility. D. Reidel, Dordrecht: Holland.

Fishburn, P.C. and LaValle, I.H. (1992) Multiattribute expected utility without the Archimedean axiom. Journal of Mathematical Pshychology 36, $573-591$.

Hausner, M. (1954) Multidimensional utilities. In R.M. Thrall, C.H. Coombs, and R.L. Davis (eds.) Decision Processes, Wiley, New York.

LaValle, I.H. and Fishburn, P.C. (1991) Lexicographic state-dependent subjective expected utility. Journal of Risk and Uncertainty 4, 251-269.

LaValle, I.H. and Fishburn, P.C. (1992) State-independent subjective expected lexicographic utility. Journal of Risk and Uncertainty 5, 217240.

Nakamura, Y. (2000) Finite-dimensional utilities. Economic Theory 16, 209-218. 